\title{
Steady Trapped Solutions to Forced Long-Short Interaction Equation
}

$\operatorname{AUTHOR}(S)$ :

Funakoshi, Mitsuaki

\section{CITATION:}

Funakoshi, Mitsuaki. Steady Trapped Solutions to Forced Long-Short Interaction Equation. 数理解析研究所講究録 1993, 830: 12-27

\section{ISSUE DATE:}

1993-04

URL:

http://hdl.handle.net/2433/83362

RIGHT: 


\title{
Steady Trapped Solutions to Forced Long-Short Interaction Equation
}

\author{
九大応力研 船越 满明 (Mitsuaki Funakoshi)
}

\section{$\S 1$. Introduction}

The resonant interaction between long and short waves is possible in several fluid systems. In a two-layer fluid system, the interaction is possible between a long internal mode and a wave packet of short surface mode whose group velocity is close to the phase speed $c_{p}^{\prime}$ of the long internal mode ${ }^{1,2)}$.

Next, if a fluid flows over an uneven bottom topography at velocity $V^{\prime}$, waves will be generated, as in the case of mountain waves in the atmosphere. For bottom topography of large horizontal scale and small height, the generation of a long wave in a single-layer fluid can be described by the forced $\mathrm{K}-\mathrm{dV}$ equation in the resonant case in which $V^{\prime}$ is close to the phase speed of this wave ${ }^{3,4)}$. The same equation is derived also for a stratified fluid system when $V^{\prime}$ is close to the phase speed of one of the wave modes of the system in the long-wave limit ${ }^{5,6}$.

In this paper, for a two-layer fluid, both the resonant interaction between the long internal mode and the short surface mode, and the generation of the long internal mode by the resonant motion of the fluid relative to the bottom topography of large horizontal scale, are examined simultaneously.

\section{$\S 2$. Forced Long-Short Interaction Equation}

We consider two-dimensional irrotational motion of a two-layer inviscid fluid over a localized bottom unevenness, as shown in Fig.1. Here the upper and lower fluids ( with densities $\rho_{1}$ and $\rho_{2}\left(>\rho_{1}\right)$ ) are assumed to be quiescent and to have depths $h_{1}$ and $h_{2}$ far from the unevenness. $\zeta_{1}^{\prime}$ and $\zeta_{2}^{\prime}$ are freesurface and interface displacements, and $b^{\prime}$ expresses the bottom unevenness. Also $x^{\prime}$ and $z^{\prime}$ are horizontal and upward vertical coordinates, and $t^{\prime}$ is the time. 
If velocity potentials of upper and lower fluids, $\phi_{1}^{\prime}$ and $\phi_{2}^{\prime}$, and $\zeta_{1}^{\prime}, \zeta_{2}^{\prime}, b^{\prime}, x^{\prime}$, $z^{\prime}, t^{\prime}$ are non-dimensionalized using $h_{1}$ and gravitational acceleration $g$, governing equations are written as

$$
\begin{aligned}
& \phi_{1 x x}+\phi_{1 z z}=0, \quad \zeta_{2}<z<1+\zeta_{1}, \\
& \left.\begin{array}{l}
\phi_{2 x x}+\phi_{2 z z}=0, \\
\zeta_{1 t}+\phi_{1 x} \zeta_{1 x}-\phi_{1 z}=0, \\
\phi_{1 t}+\zeta_{1}+\frac{1}{2}\left[\left(\phi_{1 x}\right)^{2}+\left(\phi_{1 z}\right)^{2}\right]=0,
\end{array}\right\} \text { on } z=1+\zeta_{1}, \\
& \zeta_{2 t}+\phi_{1 x} \zeta_{2 x}-\phi_{1 z}=0, \\
& \zeta_{2 t}+\phi_{2 x} \zeta_{2 x}-\phi_{2 z}=0, \\
& (1-\Delta)\left\{\phi_{1 t}+\zeta_{2}+\frac{1}{2}\left[\left(\phi_{1 x}\right)^{2}+\left(\phi_{1 z}\right)^{2}\right]\right\} \\
& -\left\{\phi_{2 t}+\zeta_{2}+\frac{1}{2}\left[\left(\phi_{2 x}\right)^{2}+\left(\phi_{2 z}\right)^{2}\right]\right\}=0, \\
& -b_{t}-b_{x} \phi_{2 x}+\phi_{2 z}=0, \quad \text { on } z=-r+b,
\end{aligned}
$$

where non-dimensionalized variables are expressed by dropping primes, and $r=h_{2} / h_{1}, \rho_{1}=\rho_{2}(1-\Delta)$.

We also assume that the bottom moves at non-dimensional speed $V$ in this reference system. Therefore, $b$ depends only on $x-V t$, and $b \rightarrow 0$ as $x-V t \rightarrow \pm \infty$. Of course, the results shown below can be used under a simple variable transformation also for the case of the uniform fluid flow at speed $-V$ over a quiescent bottom.

For flat bottom $b=0$, the linearized version of eq.(2.1) has the following two kinds of solutions :

$$
\left\{\begin{array}{l}
\zeta_{1}=a_{0} e^{\mathrm{i} \theta_{ \pm}}+\text {c.c. }, \quad \phi_{1}=\mathrm{i} a_{0} \nu_{ \pm}(z, k) e^{\mathrm{i} \theta_{ \pm}}+\text {c.c. } \\
\zeta_{2}=\mu_{ \pm}(k) a_{0} e^{\mathrm{i} \theta_{ \pm}}+\text {c.c. }, \quad \phi_{2}=\mathrm{i} a_{0} \chi_{ \pm}(z, k) e^{\mathrm{i} \theta_{ \pm}}+\text {c.c. }
\end{array}\right.
$$

where

$$
\begin{aligned}
& \theta_{ \pm}=k x-\omega_{ \pm}(k) t \\
& \omega_{ \pm}(k)=\left\{k \frac{\sigma_{1}+\sigma_{2} \pm \sqrt{\left(\sigma_{1}+\sigma_{2}\right)^{2}-4 \Delta \sigma_{1} \sigma_{2}\left[1+(1-\Delta) \sigma_{1} \sigma_{2}\right]}}{2\left[1+(1-\Delta) \sigma_{1} \sigma_{2}\right]}\right\}^{\frac{1}{2}}
\end{aligned}
$$




$$
\begin{aligned}
& \mu_{ \pm}(k)=C_{1}-\frac{k}{\omega_{ \pm}(k)^{2}} S_{1} \\
& \nu_{ \pm}(z, k)=\frac{\omega_{ \pm}(k)}{k} \sinh k(1-z)-\frac{1}{\omega_{ \pm}(k)} \cosh k(1-z), \\
& \chi_{ \pm}(z, k)=-\frac{\omega_{ \pm}(k)\left[C_{1}-k S_{1} / \omega_{ \pm}(k)^{2}\right]}{k S_{2}} \cosh k(z+r),
\end{aligned}
$$

$C_{1}=\cosh k, C_{2}=\cosh k r, S_{1}=\sinh k, S_{2}=\sinh k r, \sigma_{1}=\tanh k, \sigma_{2}=\tanh k r$.

The solution corresponding to positive sign in \pm of the above expressions is called surface mode, while that to negative sign is called internal mode.

It is well known that the surface mode of wavenumber $k_{0}$ interacts resonantly with the long internal mode if

$$
c_{g}\left(k_{0}\right)=c_{p}
$$

is satisfied, where $c_{g}(k)=d \omega_{+}(k) / d k$ is the group velocity of the surface mode, and

$$
c_{p}=\left.\frac{\omega_{-}(k)}{k}\right|_{k \rightarrow 0}=\left\{\left[1+r-\sqrt{(1+r)^{2}-4 \Delta r}\right] / 2\right\}^{\frac{1}{2}},
$$

is the phase speed of the internal mode in the long-wave limit.

Next, if the bottom is uneven and $V \approx c_{p}$, the long internal mode is generated resonantly by the unevenness. That is, since the group velocity of the right-going wave of this mode relative to the bottom is very small, the amplitude of the generated wave is expected to become much larger than the height of the bottom unevenness.

Aiming at examining the system with not only the resonant interaction but also the resonant generation, we assume that, using $\varepsilon(\ll 1)$, there exist the surface mode of wavenumber close to $k_{0}$ and of amplitude $O(\varepsilon)$ and the long internal mode of amplitude $O\left(\varepsilon^{4 / 3}\right)$ over the bottom unevenness of height $O\left(\varepsilon^{2}\right)$. Furthermore, the horizontal scales of the surface-mode wave packet, of the internal mode, and of the bottom unevenness, are assumed to be $O\left(\varepsilon^{-2 / 3}\right)$. Finally $V-c_{p}$ is assumed to be $O\left(\varepsilon^{2 / 3}\right)$. Under these assumptions, an evolution equation for the surface-mode wave packet and the long internal mode can be derived using the reductive perturbation method, as shown below. 
First, we expand the variables as

$$
\phi_{j}=\sum_{n=0}^{\infty} \varepsilon^{\frac{2+n}{3}} \phi_{j}^{(n)}, \quad \zeta_{j}=\sum_{n=0}^{\infty} \varepsilon^{\frac{2+n}{3}} \zeta_{j}^{(n)}, \quad(j=1,2),
$$

where $\phi_{j}^{(n)}$ and $\zeta_{j}^{(n)}$ are assumed to depend not only on $t, x$ and $z$ (only for $\left.\phi_{j}{ }^{(n)}\right)$ but also on a stretched horizontal coordinate $\xi=\varepsilon^{2 / 3}(x-V t)$ fixed to the bottom and a stretched time $\tau=\varepsilon^{4 / 3} t$. Next, $V$ and $b$ are expressed in the following forms :

$$
\begin{aligned}
& V=V_{0}+\varepsilon^{2 / 3} V_{1}+\cdots, \\
& b=\varepsilon^{2} H(\xi),
\end{aligned}
$$

where function $H$, satisfying $H \rightarrow 0$ as $\xi \rightarrow \pm \infty$, determines the localized bottom unevenness.

The replacements of the differential operators

$$
\frac{\partial}{\partial x} \rightarrow \frac{\partial}{\partial x}+\varepsilon^{2 / 3} \frac{\partial}{\partial \xi}, \quad \frac{\partial}{\partial t} \rightarrow \frac{\partial}{\partial t}-V \varepsilon^{2 / 3} \frac{\partial}{\partial \xi}+\varepsilon^{4 / 3} \frac{\partial}{\partial \tau},
$$

in governing eq.(2.1), substitution of expressions $(2.6) \sim(2.8)$, and the expansion of boundary conditions (2.1c) and (2.1d) around $z=1$, conditions $(2.1 \mathrm{e}) \sim(2.1 \mathrm{~g})$ around $z=0$, and condition $(2.1 \mathrm{~h})$ around $z=-r$, result in the following equations in $\mathrm{O}\left(\varepsilon^{\frac{2+n}{3}}\right)$ :

$$
\left\{\begin{array}{cc}
\phi_{1 x x}^{(n)}+\phi_{1 z z}^{(n)}=N_{1}^{(n)}, \quad 0<z<1, \\
\phi_{2 x x}^{(n)}+\phi_{2 z z}^{(n)}=N_{2}^{(n)}, \quad-r<z<0, \\
\zeta_{1 t}^{(n)}-\phi_{1 z}^{(n)}=N_{3}^{(n)}, \quad \phi_{1 t}^{(n)}+\zeta_{1}^{(n)}=N_{4}^{(n)}, \quad \text { on } z=1, \\
\zeta_{2 t}^{(n)}-\phi_{1 z}^{(n)}=N_{5}^{(n)}, \zeta_{2 t}^{(n)}-\phi_{2 z}^{(n)}=N_{6}^{(n)}, \\
(1-\Delta) \phi_{1 t}^{(n)}-\phi_{2 t}^{(n)}-\Delta \zeta_{2}^{(n)}=N_{7}^{(n)}, \quad \text { on } \quad z=0 \\
\phi_{2 z}^{(n)}=N_{8}^{(n)}, \quad \text { on } \quad z=-r,
\end{array}\right.
$$

where $n=0,1,2, \cdots$, and $N_{m}^{(n)}(m=1,2, \cdots, 8)$ depend only on the lowerorder variables in eq.(2.6), or $H$, or their derivatives.

Since $N_{m}^{(0)}=N_{m}^{(1)}=0(m=1,2, \cdots, 8)$, we can assume that

$$
\zeta_{j}^{(0)}=0, \quad \phi_{j}^{(0)}=\Phi_{j},(j=1,2),
$$




$$
\left\{\begin{array}{l}
\phi_{1}^{(1)}=\mathrm{i} A \nu_{+}\left(z, k_{0}\right) e^{\mathrm{i} \theta}+\text { c.c., } \phi_{2}^{(1)}=\mathrm{i} A \chi_{+}\left(z, k_{0}\right) e^{\mathrm{i} \theta}+\text { c.c. } \\
\zeta_{1}^{(1)}=A e^{\mathrm{i} \theta}+\text { c.c., } \zeta_{2}^{(1)}=\mu_{+}\left(k_{0}\right) A e^{\mathrm{i} \theta}+\text { c.c. } \\
\theta=k_{0} x-\omega_{+}\left(k_{0}\right) t
\end{array}\right.
$$

invoking eq.(2.2). Here $\Phi_{1}, \Phi_{2}$ and $A$ depend only on $\xi$ and $\tau$. In $\mathrm{O}\left(\varepsilon^{4 / 3}\right)$, solving eq.(2.9) with $n=2$, we obtain

$$
\left\{\begin{array}{l}
\zeta_{1}^{(2)}=V_{0} \Phi_{1 \xi}, \quad \zeta_{2}^{(2)}=\frac{V_{0}}{\Delta}\left[\Phi_{2 \xi}-(1-\Delta) \Phi_{1 \xi}\right] \\
\phi_{j}^{(2)}=\hat{\Phi}_{j}, \quad(j=1,2)
\end{array}\right.
$$

where $\hat{\Phi}_{1}$ and $\hat{\Phi}_{2}$ depend only on $\xi$ and $\tau$. Furthermore, we have

$$
\begin{aligned}
& V_{0}=c_{g}\left(k_{0}\right), \\
& V_{0}=c_{p},
\end{aligned}
$$

as the compatibility conditions in $\mathrm{O}\left(\varepsilon^{5 / 3}\right)$ and $\mathrm{O}\left(\varepsilon^{2}\right)$. Therefore, if the values of $\Delta$ and $r$ are given, wavenumber $k_{0}$ and resonance speed $V_{0}$ are determined from eqs.(2.13) and (2.14). Finally from the compatibility conditions in $O\left(\varepsilon^{7 / 3}\right)$ and $O\left(\varepsilon^{8 / 3}\right)$, we obtain an evolution equation :

$$
\left\{\begin{array}{l}
\mathrm{i} A_{\tau}-\mathrm{i} V_{1} A_{\xi}-p A_{\xi \xi}+\alpha A B=0 \\
B_{\tau}-V_{1} B_{\xi}-\beta\left(|A|^{2}\right)_{\xi}+\kappa H_{\xi}=0
\end{array}\right.
$$

where $B=\zeta_{2}^{(2)}$ and

$$
p=-\left.\frac{1}{2} \frac{d^{2} \omega_{+}(k)}{d k^{2}}\right|_{k=k_{0}}, \quad \kappa=\frac{V_{0}^{3}\left(V_{0}^{2}-1\right)}{2\left(V_{0}^{4}-\Delta r\right)} .
$$

It is easily shown that the constants $p$ and $\kappa$ are positive. The coefficients of interaction terms, $\alpha$ and $\beta$, are complicated functions of $\Delta$ and $r$, as given in Appendix. Numerical computation shows that $\alpha$ and $\beta$ are positive.

Equation (2.15) can be written in a simpler form

$$
\left\{\begin{array}{l}
\mathrm{i} S_{T}-\mathrm{i} \lambda S_{X}-S_{X X}+S L=0 \\
L_{T}-\lambda L_{X}-\left(|S|^{2}\right)_{X}+H_{X}=0
\end{array}\right.
$$

by the scale transformation

$$
S=A \sqrt{\beta / \kappa}, L=B\left(\alpha p / \kappa^{2}\right)^{1 / 3}, X=\xi\left(\kappa \alpha / p^{2}\right)^{1 / 3}, T=\tau\left(\kappa^{2} \alpha^{2} / p\right)^{1 / 3} .
$$


Here

$$
\lambda=V_{1}(\kappa \alpha p)^{-1 / 3}=\frac{V-c_{p}}{\varepsilon^{2 / 3}}(\kappa \alpha p)^{-1 / 3},
$$

expresses the small defference of $V$ from $c_{p}$.

It should be noted that eq. (2.16) for flat bottom $(H=0)$ is exactly solvable through the inverse scattering method and admits an $N$-soliton solution decaying at infinity ${ }^{7,8)}$. The one-soliton solution is

$$
\left\{\begin{aligned}
S= & \sqrt{2(\lambda+v)} s \operatorname{sech}\left[s\left(X-v T-\hat{X}_{0}\right)\right] \\
& \times \exp \left\{\mathrm{i}\left[-\frac{1}{2}(\lambda+v) X-\hat{\Omega} T+\psi_{0}\right]\right\} \\
L= & -2 s^{2} \operatorname{sech}^{2}\left[s\left(X-v T-\hat{X}_{0}\right)\right]
\end{aligned}\right.
$$

where $v, s, \hat{X}_{0}$ and $\psi_{0}$ are arbitrary constants and

$$
\hat{\Omega}=s^{2}-\frac{1}{4} v^{2}+\frac{1}{4} \lambda^{2} .
$$

Also $\lambda+v \geq 0$ must be satisfied.

\section{$\S 3$. Steady Trapped Solutions}

For localized bottom unevenness $(H \rightarrow 0$ as $X \rightarrow \pm \infty)$, we define steady trapped solutions to eq.(2.16) as those satisfying

$$
|S|=f(X), \quad L=g(X)
$$

where

$$
f(X) \rightarrow 0, \quad g(X) \rightarrow 0, \quad \text { as } \quad X \rightarrow \pm \infty .
$$

The solutions are composed of a trapped long-wave solution and a trapped coupled solution, as shown below.

\subsection{Trapped long-wave solution}

The trapped long-wave solution is expressed as

$$
S=0, \quad L=\frac{1}{\lambda} H(X) .
$$

The divergence of this solution as $\lambda \rightarrow 0$ means that the difference between $V$ and $c_{p}$ should be neither large nor too small in order that waves are described well by eq.(2.16) (For very small $\left|V-c_{p}\right|$, the adoption of a different scaling or the introduction of damping effect may be required. ). Hereafter, we assume that $\lambda$ is non-zero. 


\subsection{Trapped coupled solution}

We assume that

$$
S=f(X) \exp \{\mathrm{i}[\varphi(X)-\Omega T]\}, \quad L=g(X),
$$

where $f, \varphi$ and $g$ are real functions and $\Omega$ is a real constant. Only the $T$ dependence given in eq.(3.4) is allowed by eq.(2.16). Substituting eq.(3.4) into eq.(2.16), we have

$$
\left\{\begin{array}{l}
\left(2 \varphi^{\prime}+\lambda\right) f^{\prime}+\varphi^{\prime \prime} f=0 \\
f^{\prime \prime}-\left(\Omega+\varphi^{\prime 2}+\lambda \varphi^{\prime}\right) f-f g=0 \\
f^{2}-H+\lambda g=0
\end{array}\right.
$$

invoking eq.(3.2), where primes denote differentiation with respect to $X$. Equation (3.5a) can be integrated to give

$$
f^{2}\left(\varphi^{\prime}+\frac{1}{2} \lambda\right)=a
$$

where $a$ is a constant. If $a \neq 0$, the condition (3.2) demands that $\varphi^{\prime} \rightarrow \pm \infty$ as $X \rightarrow \pm \infty$. Since this situation contradicts with the assumption that surfacemode wavenumber is close to $k_{0}$, we choose that $a=0$. Therefore, we have

$$
\varphi(X)=-\frac{1}{2} \lambda X+X_{0},
$$

where $X_{0}$ is a constant. Substituting eqs.(3.5c) and (3.6) into eq.(3.5b), we obtain an equation for $f$ :

$$
f^{\prime \prime}+\frac{1}{\lambda} f^{3}+\left(-\frac{1}{\lambda} H+\frac{1}{4} \lambda^{2}-\Omega\right) f=0 .
$$

Boundary condition (3.2) and eq.(3.7) produce an eigenvalue problem with eigenvalue $\Omega$ for specified function $H$ and value $\lambda$. Here it should be noted that $\Omega$ must satisfy the condition

$$
\Omega>\frac{1}{4} \lambda^{2}
$$

because the main terms in eq.(3.7) at infinity are

$$
f^{\prime \prime}+\left(\frac{1}{4} \lambda^{2}-\Omega\right) f=0
$$




\section{§4. Trapped Coupled Solutions for Sech ${ }^{2}$-Type Bottom Unevenness}

Hereafter, we restrict our study to the following symmetric bottom unevenness :

$$
H=\alpha \operatorname{sech}^{2} q X,
$$

where $\alpha=1$ (corresponding to a mountain) or $\alpha=-1$ (a valley), and $q$ is a positive constant.

\subsection{Analytic solutions}

Equations (3.7) and (3.5c) with $H$ given in eq.(4.1) have a symmetric analytic solution

$$
\left\{\begin{array}{l}
f=\sqrt{\alpha+2 \lambda q^{2}} \operatorname{sech} q X \\
\Omega=\frac{1}{4} \lambda^{2}+q^{2} \\
g=-2 q^{2} \operatorname{sech}^{2} q X
\end{array}\right.
$$

if $\lambda$ satisfies $\alpha+2 \lambda q^{2} \geq 0$.

Next, if $f$ is much smaller than 1 for all $X$, the nonlinear term in eq.(3.7) can be neglected. Then eq.(3.7) with eq.(4.1) is transformed into the Legendre's associated differential equation :

$$
\left(1-\eta^{2}\right) \frac{d^{2} f}{d \eta^{2}}-2 \eta \frac{d f}{d \eta}+\left[\nu(\nu+1)-\frac{\mu^{2}}{1-\eta^{2}}\right] f=0,
$$

where $\eta=\tanh q X$, and

$$
\nu(\nu+1)=-\frac{\alpha}{\lambda q^{2}}, \quad \mu^{2}=\frac{1}{q^{2}}\left(\Omega-\frac{1}{4} \lambda^{2}\right) .
$$

Here we assume that $\mu>0$ without loss of generality. Symmetric localized solutions to eq.(4.3) exist only when

$$
\mu=\nu-2 N+2>0, \quad(N=1,2, \cdots),
$$

is satisfied, whereas antisymmetric ones exist only when

$$
\mu=\nu-2 N+1>0, \quad(N=1,2, \cdots),
$$

is satisfied. We refer to these solutions for each $N$ as the $N$-th symmetric and antisymmetric modes. The $N$-th (symmetric or antisymmetric) mode 
has $N-1$ positive zeros if $\mu$ and $\nu$ are integers. This seems to be true also for non-integer $\mu$ and $\nu$. Using eq.(4.4) in condition (4.5), we find that the first symmetric mode exists for all $\lambda$ satisfying $\lambda / \alpha<0$, while the $N$-th symmetric mode $(N \geq 2)$ exists only for

$$
0>\frac{\lambda}{\alpha} \geq-\frac{1}{2 q^{2}(2 N-1)(N-1)} .
$$

Whereas, eqs.(4.4) and (4.6) give that the $N$-th antisymmetric mode $(N \geq 1)$ exists only for

$$
0>\frac{\lambda}{\alpha} \geq-\frac{1}{2 q^{2} N(2 N-1)}
$$

Therefore, more (symmetric or antisymmetric) modes are found for smaller positive $-\lambda / \alpha$. Moreover, the $\Omega$ values for the $N$-th symmetric and antisymmetric modes are

$$
\Omega=\frac{1}{4} \lambda^{2}-\frac{\alpha}{\lambda}+\frac{1}{2} q^{2}\left(8 N^{2}-12 N+5\right)-q(4 N-3) \sqrt{\frac{1}{4} q^{2}-\frac{\alpha}{\lambda}},
$$

and

$$
\Omega=\frac{1}{4} \lambda^{2}-\frac{\alpha}{\lambda}+\frac{1}{2} q^{2}\left(8 N^{2}-4 N+1\right)-q(4 N-1) \sqrt{\frac{1}{4} q^{2}-\frac{\alpha}{\lambda}},
$$

respectively.

\subsection{Method of numerical computation}

The trapped coupled solutions for $q=1$ were computed numerically. That is, solutions to the equation

$$
f^{\prime \prime}+\frac{1}{\lambda} f^{3}+\left(-\frac{\alpha}{\lambda} \operatorname{sech}^{2} X+\frac{1}{4} \lambda^{2}-\Omega\right) f=0
$$

satisfying $f \rightarrow 0$ as $X \rightarrow \pm \infty$, were computed for each $\lambda, \alpha$ and $\Omega$ values by the following method. We first assume that $f$ approximately satisfies eq.(3.9) for $X \geq X_{\mathrm{e}}>0$, using a large constant $X_{\mathrm{e}}$. Then we obtain

$$
f=\nu_{0} \exp \left(-\sqrt{\Omega-\lambda^{2} / 4} X\right), \quad \text { for } X \geq X_{\mathrm{e}},
$$

where $\nu_{0}$ is a constant. If we integrate eq.(4.11) from $X=X_{\mathrm{e}}$ to $X=0$ with the initial condition computed from eq.(4.12), we have $f(0)$ and $f^{\prime}(0)$ values for each $\nu_{0}$ (Hereafter, we refer to $f(0)$ and $f^{\prime}(0)$ as $f_{0}$ and $f_{0}^{\prime}$ ). Therefore, 
by computing them for a wide range of $\nu_{0}$, we obtain a curve drawn on the $\left(f_{0}, f_{0}^{\prime}\right)$ plane. We call this the $\left(f_{0}, f_{0}^{\prime}\right)$ curve.

Next, if there is a trapped coupled solution, it can be considered as the connection of a solution $f_{p}(X)$ for $X \geq 0$ and a solution $f_{n}(X)$ for $X \leq 0$. The connection conditions to be satisfied are that $f_{p}(0)=f_{n}(0)$ and $f_{p}^{\prime}(0)=f_{n}^{\prime}(0)$. Here, if $f(X)$ is a solution to eq. $(4.11)$, then $f(-X)$ and $-f(-X)$ are also solutions. Therefore, $f_{r 1}(X) \equiv f_{n}(-X)$ and $f_{r 2}(X) \equiv-f_{n}(-X)$ are also the solutions for $X \geq 0$, and they satisfy $f_{r 1}(0)=f_{p}(0), f_{r 1}^{\prime}(0)=-f_{p}^{\prime}(0)$, and $f_{r 2}(0)=-f_{p}(0), f_{r 2}^{\prime}(0)=f_{p}^{\prime}(0)$. Consequently, the intersection between the original $\left(f_{0}, f_{0}^{\prime}\right)$ curve and either of the $\left(f_{0},-f_{0}^{\prime}\right)$ curve and the $\left(-f_{0}, f_{0}^{\prime}\right)$ curve implies the existence of a trapped coupled solution. Among the intersections, those satisfying $f_{0}^{\prime}=0$ correspond to symmetric solutions, whereas those satisfying $f_{0}=0$ correspond to antisymmetric ones. Other intersections with $f_{0} \neq 0$ and $f_{0}^{\prime} \neq 0$ imply the solutions which are neither symmetric nor antisymmetric. Hereafter, we refer to the solutions as asymmetric solutions.

In the numerical computations, Adams predictor-corrector method was used to integrate eq.(4.11). Here $X_{\mathrm{e}}$ was determined as the minimum value satisfying both

$$
\frac{\nu_{0}^{2}}{|\lambda|} \exp \left[-2 \sqrt{\Omega-\lambda^{2} / 4} X_{\mathrm{e}}\right] \leq \varepsilon_{1}\left(\Omega-\frac{1}{4} \lambda^{2}\right)
$$

and

$$
\frac{4}{|\lambda|} \exp \left(-2 X_{\mathrm{e}}\right) \leq \varepsilon_{2}\left(\Omega-\frac{1}{4} \lambda^{2}\right)
$$

for small $\varepsilon_{1}$ and $\varepsilon_{2}$. Here $\varepsilon_{1}=\varepsilon_{2}=10^{-8}$ was usually used, and the accuracy was checked by comparing with the result for $\varepsilon_{1}$ and $\varepsilon_{2} \quad 10^{-1}$ times smaller than the previous value.

4.3 Solutions for negative unevenness $(\alpha=-1)$

4.3.1 The case of $\lambda>0$

For $\lambda>0$, in addition to symmetric solutions, antisymmetric and asymmetric solutions are obtained. Figure 2 shows the $\left(\sqrt{f_{0}^{2}+f_{0}^{\prime 2}}, \Omega\right)$ values of these solutions for $\lambda=0.5$. The solutions on the main branches of symmetric and antisymmetric solutions, expressed by $S_{1}$ and $A_{1}$, asymptote to the first symmetric and antisymmetric modes introduced in $\S 4.1$ in the limit $\sqrt{f_{0}^{2}+f_{0}^{\prime 2}} \rightarrow 0$, respectively. Also the $\Omega$ values in this limit agree with those 
computed from eqs.(4.9) and (4.10) with $N=1$ and $q=1$. Moreover, these solutions have no positive zero even for non-small $\sqrt{f_{0}^{2}+f_{0}^{\prime 2}}$, similarly to these modes. As $\sqrt{f_{0}^{2}+f_{0}^{\prime 2}}$ increases, a few subbranches appear near the main branches. Most of them are always close to the main branches. The solutions on the subbranches are close to the superposition of the solutions on the main branches and one or more one-soliton solutions (2.18) for flat bottom with $v=0$ and $\hat{\Omega}=\Omega$, as shown in the inlets of Fig.2. Two subbranches of asymmetric solutions, however, part from the main branches as $\sqrt{f_{0}^{2}+f_{0}^{\prime 2}}$ increases. The solutions on this part of these branches are quite different from symmetric or antisymmetric solutions near $X=0$.

Figure 3 shows the solutions for smaller $\lambda(\lambda=0.07)$. The solutions on branches $S_{1}$ and $S_{2}$ asymptote to the first and second symmetric modes in the limit $\sqrt{f_{0}^{2}+f_{0}^{\prime 2}} \rightarrow 0$, whereas those on $\mathrm{A}_{1}$ and $\mathrm{A}_{2}$ to the first and second antisymmetric modes. Moreover, even for non-small $\sqrt{f_{0}^{2}+f_{0}^{\prime 2}}$, the solutions on branches $S_{1}$ and $A_{1}$ have no zero, and those on $S_{2}$ and $A_{2}$ have one zero, similarly to the corresponding modes. Although there exist many subbranches near these four main branches, they are not shown in Fig. 3 as long as they are close to the main branches. Only an asymmetric subbranch diverging from $\mathrm{S}_{2}$ is shown. Moreover, a symmetric branch $\mathrm{S}_{2,3}$ is found just below $\mathrm{S}_{2}$. The solutions on this branch are not related to the symmetric mode. They have two non-small peaks at $X$ far from 0 , as shown in the inlets. The peaks move to $X= \pm \infty$; as $f_{0}$ tends to 0 .

As illustrated in Figs.2 and 3, for each $\lambda$, the numbers of symmetric and antisymmetric main branches and the asymptotic $\Omega$ values of these branches as $\sqrt{f_{0}{ }^{2}+f_{0}^{\prime 2}} \rightarrow 0$ can be predicted from the localized solutions to eq.(4.3) (except for the branches such as $\mathrm{S}_{2,3}$ ).

4.3.2 The case of $\lambda<0$

For negative unevenness with $\lambda<0$, we find no solutions. This is consistent with the non-existence of the localized solution to eq.(4.3) for $\lambda / \alpha>0$. 4.4 Solutions for positive unevenness $(\alpha=1)$

4.4.1 The case of $\lambda<0$

For each negative $\lambda$, one or more branches of symmetric or antisymmetric solutions are found. The number of branches and the $\Omega$ value in the limit 
$\sqrt{f_{0}^{2}+f_{0}^{\prime 2}} \rightarrow 0$ can be predicted from the solutions to eq.(4.3), as in the case of negative unevenness with $\lambda>0$. Since $\lambda$ appears in the form $\lambda / \alpha$ in eqs.(4.7) and (4.8), the range of $-\lambda$ for the existence of each mode for positive unevenness is the same as the range of $\lambda$ for negative unevenness. Therefore, as in $\S 4.3 .1$, more branches are found for smaller $|\lambda|$.

The $\left(f_{0}, \Omega\right)$ values of the solutions for fairly large $|\lambda|(\lambda=-3)$ are shown in Fig.4. Only one symmetric branch $S_{1}$ is obtained for this $\lambda$. The solution asymptotes to the first symmetric mode as $f_{0} \rightarrow 0$. Whereas, unlike the case of negative unevenness with $\lambda>0, \Omega$ decreases with the increase of $f_{0}$. Then the branch finishes by arriving at $\Omega=\lambda^{2} / 4$, where the solution cease to decay exponentially as $X \rightarrow \pm \infty$. An example of the slow-decay solution is shown in the inlet of Fig.4.

For smaller $|\lambda|$, more branches are found, as illustrated in Fig.5. Figure 5 shows the existence of three symmetric branches $\left(S_{1}, S_{2}\right.$ and $\left.S_{3}\right)$ and two antisymmetric branches $\left(A_{1}\right.$ and $\left.A_{2}\right)$ for $\lambda=-1 / 30$. The number of zeros of the solution on each branch is the same as that of the corresponding mode. All branches have smaller $\Omega$ values for larger $\sqrt{f_{0}^{2}+f_{0}^{\prime 2}}$ and finish at $\Omega=\lambda^{2} / 4$. Analytic solution (4.2) is located on branch $S_{1}$.

For positive unevenness with $\lambda<0$, the solution similar to those on the subbranches in Fig. 2 is not found. This is probably because the one-soliton solution (2.18) with $v=0$ does not exist for $\lambda<0$. Moreover, no asymmetric solution is obtained in this case.

4.4.2 The case of $\lambda>0$

For positive unevenness with $\lambda>0$, symmetric and asymmetric solutions are found, although eq.(4.3) has no localized solution for $\lambda / \alpha>0$. Antisymmetric solution is not obtained in this case. Figure 6 shows the $\left(f_{0}, \Omega\right)$ values of the solutions for fairly small $\lambda(\lambda=0.2)$. There is a main branch $\mathrm{S}_{\mathrm{M}}$ whose $\Omega$ value decreases at first and then increases as $f_{0}$ increases. Analytic solution (4.2) is on this branch. The solutions on $\mathrm{S}_{M}$ have only one peak for $f_{0}>1.13$, whereas they have two peaks for $f_{0}<1.13$. There are also subbranches of symmetric and asymmetric solutions near $\mathrm{S}_{\mathrm{M}}$, as shown in Fig.6. The solutions on these subbranches are close to the superposition of the solution on $S_{M}$ and one or more one-soliton solutions (2.18) for flat bottom with $v=0$ and 
$\hat{\Omega}=\Omega$, like the case of negative unevenness with $\lambda>0$. However, unlike this case, the peaks at $X$ far from 0 always have the same sign as the central part of the solutions, as shown in the inlets of Fig.6. In addition to branch $S_{M}$ and its subbranches, there are branches of asymmetric solutions connecting the right-hand and the left-hand parts of $S_{M}$. These branches may be associated with their subbranches. Moreover, the solutions on the central part of these branches are quite different from those on branch $S_{M}$ or its subbranches.

\section{$\S 5$. Conclusions}

In a two-layer fluid system, the forced long-short interaction equation (2.15) describes both the resonant interaction between long internal mode and short surface mode, and the wave generation by the resonant motion of the fluid relative to a bottom topography of large horizontal scale. For localized symmetric bottom unevenness of sech $^{2}$-type, this equation has coupled solutions corresponding to steady waves trapped by the unevenness. When the unevenness is negative, the steady trapped solutions, computed as the localized solutions to eq.(3.7), are found only for $\lambda>0$. The solutions are composed of one or more branches of symmetric, antisymmetric, or asymmetric (neither symmetric nor antisymmetric) solutions. Some of them have many peaks in short-wave envelope $f$. For positive unevenness, steady trapped solutions exist both for $\lambda>0$ and $\lambda<0$. The solutions for $\lambda>0$ are either symmetric or asymmetric. Moreover, a variety of solutions with many peaks in $f$ are obtained for this case. The solutions for $\lambda<0$ are composed of one or more branches of symmetric or antisymmetric solutions. For positive unevenness with $\lambda<0$ or for negative unevenness with $\lambda>0$, the range of $\lambda$ for the existence of each branch of the steady trapped solutions is partly predicted by the solutions to the linearized eq.(4.3).

The one-soliton solution (2.18) for flat bottom can be thought as a steady progressive wave far from the localized bottom unevenness. Moreover, $S=$ $0, L=\psi(X+\lambda T)$ ( $\psi$ is an arbitrary function), the solution to eq.(2.16) with $H \equiv 0$, is also a steady progressive wave far from the bottom unevenness. The interaction between the steady trapped waves shown in this paper and these two kinds of progressive waves coming to the bottom unevenness may be an interesting theme. Its characteristics will be shown in the near future. 
Appendix

The constants $\alpha$ and $\beta$ in eq.(2.15) are given by

$$
\begin{aligned}
\alpha= & k_{0} \hat{\omega}\left\{\Delta\left(1-k_{0}{ }^{2} / \hat{\omega}^{4}\right)\left(\hat{\sigma}_{2}-\hat{\sigma}_{1}\right)+2 \Delta\left(k_{0} \hat{\sigma}_{1} / \hat{\omega}^{2}-1\right) / \hat{\omega} V_{0}\right. \\
& +V_{0}^{2}\left[\left(1-k_{0}{ }^{2} / \hat{\omega}^{4}\right)\left(\hat{\sigma}_{1}+\hat{\sigma}_{2}-\Delta \hat{\sigma}_{2}\left(1+k_{0} \hat{\sigma}_{1} / \hat{\omega}^{2}\right)\right)\right. \\
& \left.-2\left((1-\Delta)\left(1+\hat{\sigma}_{1} \hat{\sigma}_{2}\right)+\Delta k_{0} \hat{\sigma}_{1}\left(1-k_{0} \hat{\sigma}_{2} / \hat{\omega}^{2}\right) / \hat{\omega}^{2}\right) / \hat{\omega} V_{0}\right] \\
& \left./\left(V_{0}^{2}-1\right)\right\} /\left[4\left(1+(1-\Delta) \hat{\sigma}_{1} \hat{\sigma}_{2}\right)-2 k_{0}\left(\hat{\sigma}_{1}+\hat{\sigma}_{2}\right) / \hat{\omega}^{2}\right] \\
\beta= & -r\left\{(1-\Delta) V_{0}\left(\hat{\omega}^{2}-k_{0}{ }^{2} / \hat{\omega}^{2}\right)-2(1-\Delta) k_{0} V_{0}^{2} / \hat{\omega}\right. \\
& -2 \hat{\omega} V_{0}^{2}(1-\Delta)\left(\hat{C}_{1}-k_{0} \hat{S}_{1} / \hat{\omega}^{2}\right)\left(\hat{S}_{1}-k_{0} \hat{C}_{1} / \hat{\omega}^{2}\right) \\
& \left.-\left[\hat{\omega}^{2} V_{0}\left(V_{0}^{2}-1\right)\left(1 / \hat{\sigma}_{2}{ }^{2}-1\right)+2 \hat{\omega}\left(V_{0}^{2}-\Delta\right) / \hat{\sigma}_{2}\right]\left(\hat{C}_{1}-k_{0} \hat{S}_{1} / \hat{\omega}^{2}\right)^{2}\right\} \\
& / 2\left(\Delta r-V_{0}^{4}\right),
\end{aligned}
$$

where $\hat{\omega}=\omega_{+}\left(k_{0}\right), \hat{C}_{1}=\cosh k_{0}, \hat{S}_{1}=\sinh k_{0}, \hat{\sigma}_{1}=\tanh k_{0}, \hat{\sigma}_{2}=\tanh k_{0} r$.

\section{References}

1) M. Funakoshi and M. Oikawa : J. Phys. Soc. Jpn. 52 (1983) 1982.

2) M. Oikawa, M. Okamura and M. Funakoshi : J. Phys. Soc. Jpn. 58 (1989) 4416.

3) T. Y. Wu : J. Fluid Mech. 184 (1987) 75.

4) J. Kevorkian and J. Yu : J. Fluid Mech. 204 (1989) 31.

5) R. H. J. Grimshaw and N. Smyth : J. Fluid Mech. 169 (1986) 429.

6) S. S. Shen : J. Fluid Mech. 234 (1992) 583.

7) N. Yajima and M. Oikawa : Prog. Theor. Phys. 56 (1976) 1719.

8) Y.-C. Ma : Studies Appl. Math. 59 (1978) 201. 


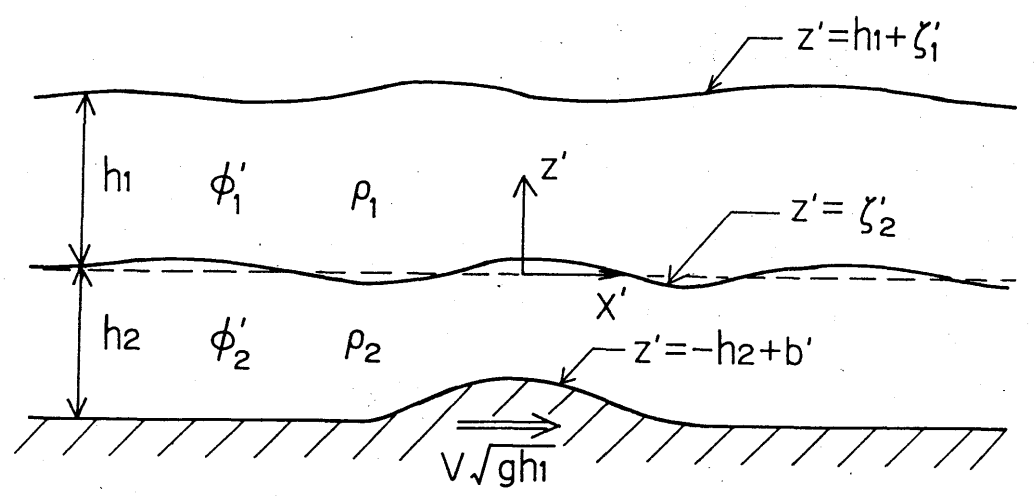

Figure 1. Schematic diagram of a two-layer fluid over uneven bottom.

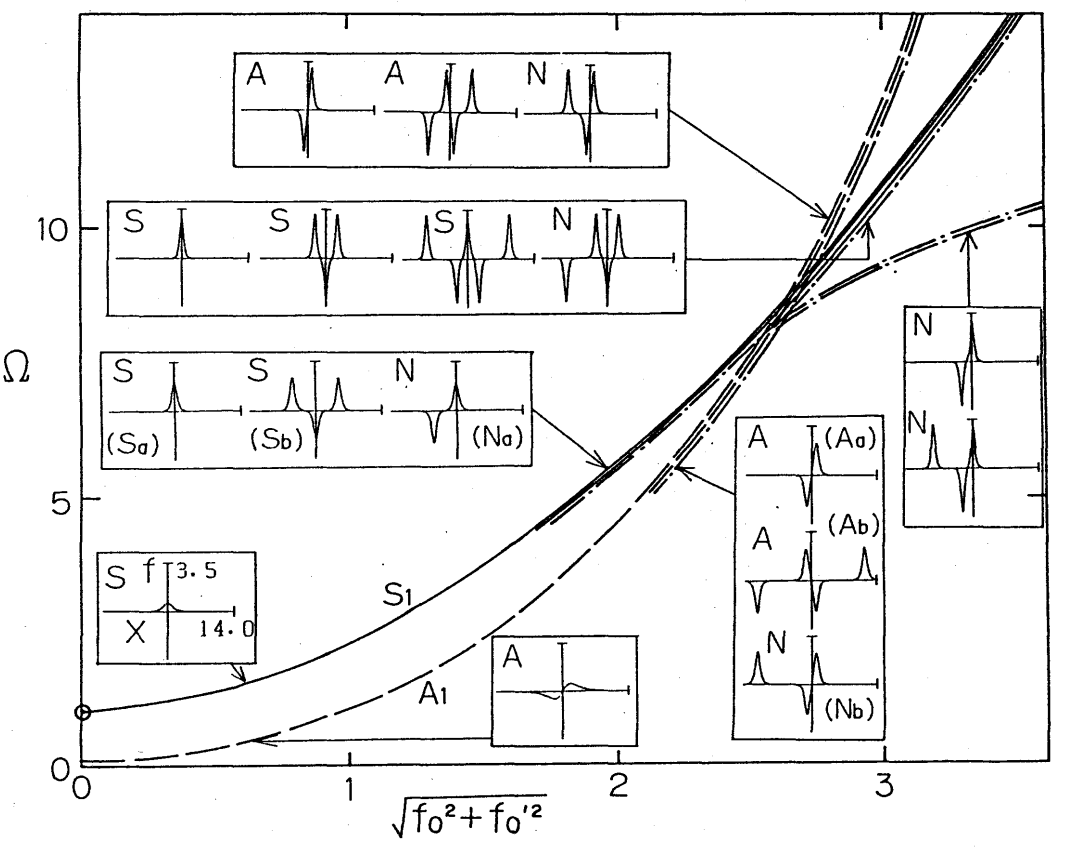

Figure 2.

$\left(\sqrt{f_{0}^{2}+f_{0}^{\prime 2}}, \Omega\right)$ values of trapped solutions for $\alpha=-1$ and $\lambda=$ 0.5 . - : symmetric solutions ( $\mathrm{S}$ in inlets), - - : antisymmetric solutions (A), -. - - - : asymmetric solutions $(\mathrm{N})$. Several typical solutions are shown in inlets. $O$ : analytic solution (4.2).

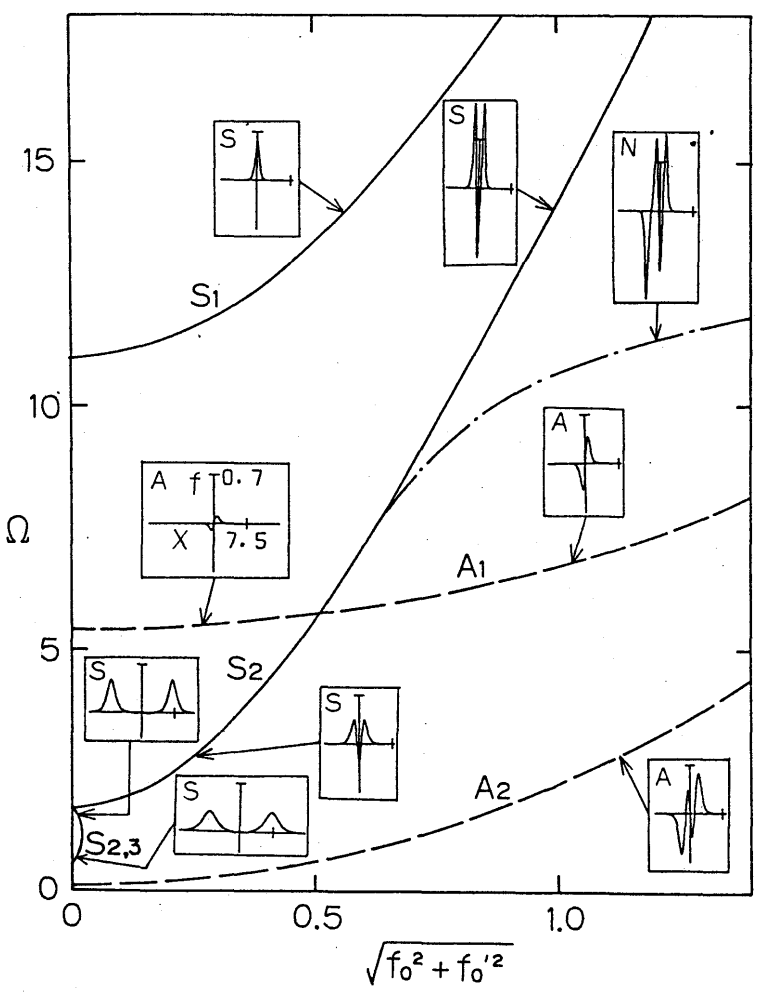

Figure 3. $\left(\sqrt{f_{0}^{2}+f_{0}^{\prime 2}}, \Omega\right)$ values of trapped solutions for $\alpha=-1$ and $\lambda=0.07$. : : symmetric ( $\mathrm{S}$ in inlets), - antisymmetric (A), - - - - - : asymmetric $(\mathrm{N})$. Several typical solutions are shown in inlets. 

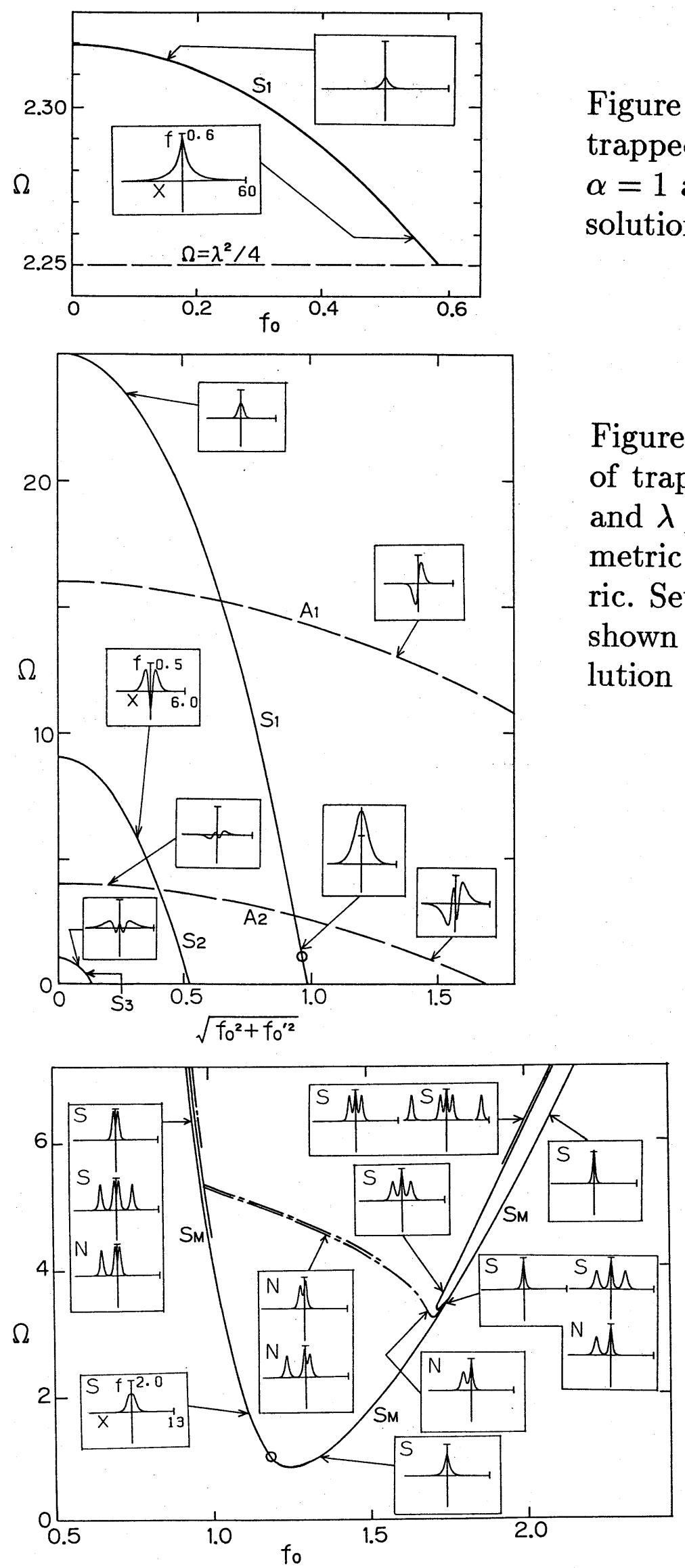

Figure 4. $\left(f_{0}, \Omega\right)$ values of trapped symmetric solutions for $\alpha=1$ and $\lambda=-3$. Two typical solutions are shown in inlets.

Figure 5. $\left(\sqrt{f_{0}^{2}+f_{0}^{\prime 2}}, \Omega\right)$ values of trapped solutions for $\alpha=1$ and $\lambda=-1 / 30$. : symmetric, - - : antisymmetric. Several typical solutions are shown in inlets. $O$ : analytic solution (4.2).
Figure 6. $\left(f_{0}, \Omega\right)$ values of trapped solutions for $\alpha=1$ and $\lambda=0.2$.

- : symmetric ( $\mathrm{S}$ in inlets), -.—. : asymmetric (N). Several typical solutions are shown in inlets. $O$ : analytic solution (4.2). 